\title{
Quantitative analyses of the 3D nuclear landscape recorded with super- resolved fluorescence microscopy
}

\author{
Volker J Schmid ${ }^{\mathrm{a} *}$, Marion Cremer $^{\mathrm{b}}$, Thomas Cremer ${ }^{\mathrm{b}}$ \\ a Biolmaging group, Department of Statistics, Ludwig Maximilians-Universität München, Ludwigstrasse 33 , 80539 Munich, \\ Germany, volker.schmid@Imu.de \\ b Biocenter, Department Biology II, Ludwig Maximilians-Universität München, Großhadernerstrasse 2, 82152 Martinsried, \\ Germany, Marion.Cremer@Irz.uni-muenchen.de, Thomas.Cremer@Irz.uni-muenchen.de
}

Preprint. Final version will be published in Methods, available at https://dx.doi.org/10.1016/j.ymeth.2017.03.013.

\begin{abstract}
Recent advancements of super-resolved fluorescence microscopy have revolutionized microscopic studies of cells, including the exceedingly complex structural organization of cell nuclei in space and time. In this paper we describe and discuss tools for (semi-) automated, quantitative 3D analyses of the spatial nuclear organization. These tools allow the quantitative assessment of highly resolved different chromatin compaction levels in individual cell nuclei, which reflect functionally different regions or sub-compartments of the 3D nuclear landscape, and measurements of absolute distances between sites of different chromatin compaction. In addition, these tools allow 3D mapping of specific DNA/RNA sequences and nuclear proteins relative to the 3D chromatin compaction maps and comparisons of multiple cell nuclei. The tools are available in the free and open source $\mathrm{R}$ packages nucim and bioimagetools. We discuss the use of masks for the segmentation of nuclei and the use of DNA stains, such as DAPI, as a proxy for local differences in chromatin compaction. We further discuss the limitations of $3 \mathrm{D}$ maps of the nuclear landscape as well as problems of the biological interpretation of such data.
\end{abstract}

\section{KEYWORDS}

super-resolution fluorescence microscopy; nucleome; chromatin compaction maps; quantitative image analysis; 3D nuclear topography of DNA, RNA and proteins

\section{ABREVIATIONS}

3D-SIM, 3D structured illumination microscopy;

ANC, active nuclear compartment;

$\mathrm{CD}(\mathrm{C})$, chromatin domain (cluster);

$\mathrm{CT}$, chromosome territory;

IC, interchromatin compartment;
DAPI, 4,6-Diamidino-2-phenylindole, dihydrochloride;

INC, inactive nuclear compartment;

$\mathrm{PR}$, perichromatin region;

$T A D$, topologically associated domain. 


\section{INTRODUCTION}

This contribution deals with 3D image analyses of nuclei to quantitatively assess 3D nuclear landscapes shaped by different chromatin compaction levels and assigning specific nuclear targets on chromatin compaction maps.

\section{Biological background}

Gene regulation, the replication of DNA and epigenetic markers, as well as the maintenance of genome integrity depend on an exceedingly complex structural nuclear organization [1]. The spacetime implications of this dynamic nuclear landscape for nuclear functions have become the focus of a new research field, called the 4D nucleome [2]. Microscopic investigations have demonstrated a structural organization of chromosome territories (CTs) built up from 1 Mb chromatin domains (CDs) $[3,4],[5]$. CDs are considered as fundamental subunits of chromatin organization with important functional implications, including the spatially defined action of regulatory sequences [1]. They are composed of smaller subdomains and form higher order structures, called chromatin domain clusters (CDCs), which constitute a spongiform chromatin network expanding throughout the nucleus. This chromatin network is co-aligned with a channel-like network starting at nuclear pores, called the interchromatin compartment (IC). IC-channels expand throughout CTs and extend with fine branches also into the CDC interior. More bulky IC-lacunas carry non-chromatin nuclear domains, such as splicing speckles, which serve as storage and assembly sites for factors and factor assemblies, required for nuclear functions. Recent studies demonstrated that CDCs show a shell-like layered organization with a peripheral layer of low chromatin density, called the perichromatin region (PR), while additional chromatin layers with increasing compaction are located in the CDC interior. Numerous studies have pointed to the PR as the chromatin subcompartment, where transcription, cotransitional splicing, DNA/chromatin replication and repair preferentially occur, although loops of chromatin or DNA may penetrate into the IC as well. Based on these observations, the IC and the PR have been considered as the active nuclear compartment (ANC), whereas the inactive nuclear compartment (INC) relates to compact chromatin in the interior of CDCs [4]. Biochemical approaches, such as $\mathrm{Hi}-\mathrm{C}$, provided independent evidence for $\sim 1 \mathrm{Mb}$ chromatin domains, called topologically associating domains (TADs) [6]. Similar to CDs and CDCs, TADs are built up from smaller domains and form larger arrangements, called metaTADs [7].

\section{Image acquisition and image analysis}

Essential progress in microscopic studies of the nuclear landscape and their dynamics was strongly facilitated by the implementation of super-resolved fluorescence microscopy of fixed and more recently also of living cells [8]. Super-resolved images require appropriate and elaborate methods of quantitative 3D image analysis. Quantitative imaging of fixed nuclei provides 3D snap-shots from individual nuclei and serial snap-shots can provide insights into the space-time dynamics of nuclear organization. Quantitative 4D image analysis to study such dynamics directly in living cells requires even more sophisticated methods which are beyond the scope of this paper. Quantitative analysis of light optical serial sections recorded from a given nucleus include segmentation of regions of interest in each optical section or simultaneously in the entire image stack [9]. Several methods for segmentation have been proposed, which have to be chosen appropriately for the aim of the 
segmentation. For example, Gaussian mixture models can be used for unsupervised segmentation [10]. The approach described below starts with the identification of a given nucleus and its 3D border within an image stack, followed by the identification of regions with distinctly different chromatin compaction. For reliable quantitative results, the analysis of a series of nuclei is required. Statistical analysis of imaging studies is frequently used in medical imaging [11], where standard protocols exist for both imaging and statistical analysis, but is still less prominent in microscopy imaging. Hence, there is a need to "pipeline" the quantitative analysis of super-resolved microscopic images of nuclear landscapes.

Software packages for image processing and visualization have been developed by commercial providers, such as Volocity, Imaris, Metamorph or Cell Profiler. These packages are usually rather expensive and did not fully fit the needs of our own studies. Free open source software has been made available by the scientific community as a cheap and reliable alternative. Open source software for microscopic imaging is typically based on Java (e.g. ImageJ and Icy), Python (BioimageXD), or R (EBImage). Using a command line based programming language allows to automate imaging processing and analysis, which is important for the analysis of multiple images. The workflow described in this paper is written in $R$. $R$ is a software for statistical computing and graphics [12]. It is available free on Windows, macOS and a variety of Linux and other UNIX systems. $R$ is command line based, but graphical front ends like RStudio [13] are available. $\mathrm{R}$ can be expanded by using additional packages, with over 9300 packages currently available on the official "Comprehensive R Archive Network" (CRAN). This allows us to easily use statistical methods for additional analysis after image processing.

\section{Outline}

Below we focus on two related issues of image analyses. Firstly, we describe and discuss tools for a semi-automated, quantitative $3 \mathrm{D}$ analysis of the spatial organization of a range of chromatin compaction levels constituting higher order chromatin landscapes. Firstly, intensity of DAPI staining of nuclear DNA is employed as a proxy for chromatin compaction. Based on pragmatic reasons and convenience seven DAPI intensity classes are distinguished for this purpose. In addition, we describe an approach for measurements of minimal absolute distances between DAPI intensity classes. Secondly, we show how markers of interest, such as specific nucleic acid sequences or functionally important proteins or protein complexes can be quantitatively assigned on the respective intensity classes that have previously been shown to reflect subcompartments of the 3D nuclear landscape with different functional assignment $[14,15,16]$.

\section{METHODS and RESULTS}

We exemplify our approach using a 3D image stack comprising a complete DAPI stained nucleus from fixed, cultured cells recorded by 3D-structured illumination microscopy (3D-SIM). The workflow of the entire approach is shown in Fig. 1. It should be emphasized, that this approach can in principle be applied for other DNA super-resolution microscopic approaches as well. 3D-SIM allows optical sectioning with a resolution of $\sim 120 \mathrm{~nm}$ lateral and 250-300 nm axial (for review see [17]). Depending on the thickness of nuclei $(\sim 5-10 \mu \mathrm{m})$ a 3D-SIM image stack contains about $40-80$ sequential SIM sections at z-distances of $125 \mathrm{~nm}$. For the recording of appropriate 3D imaging of nuclei and control of 


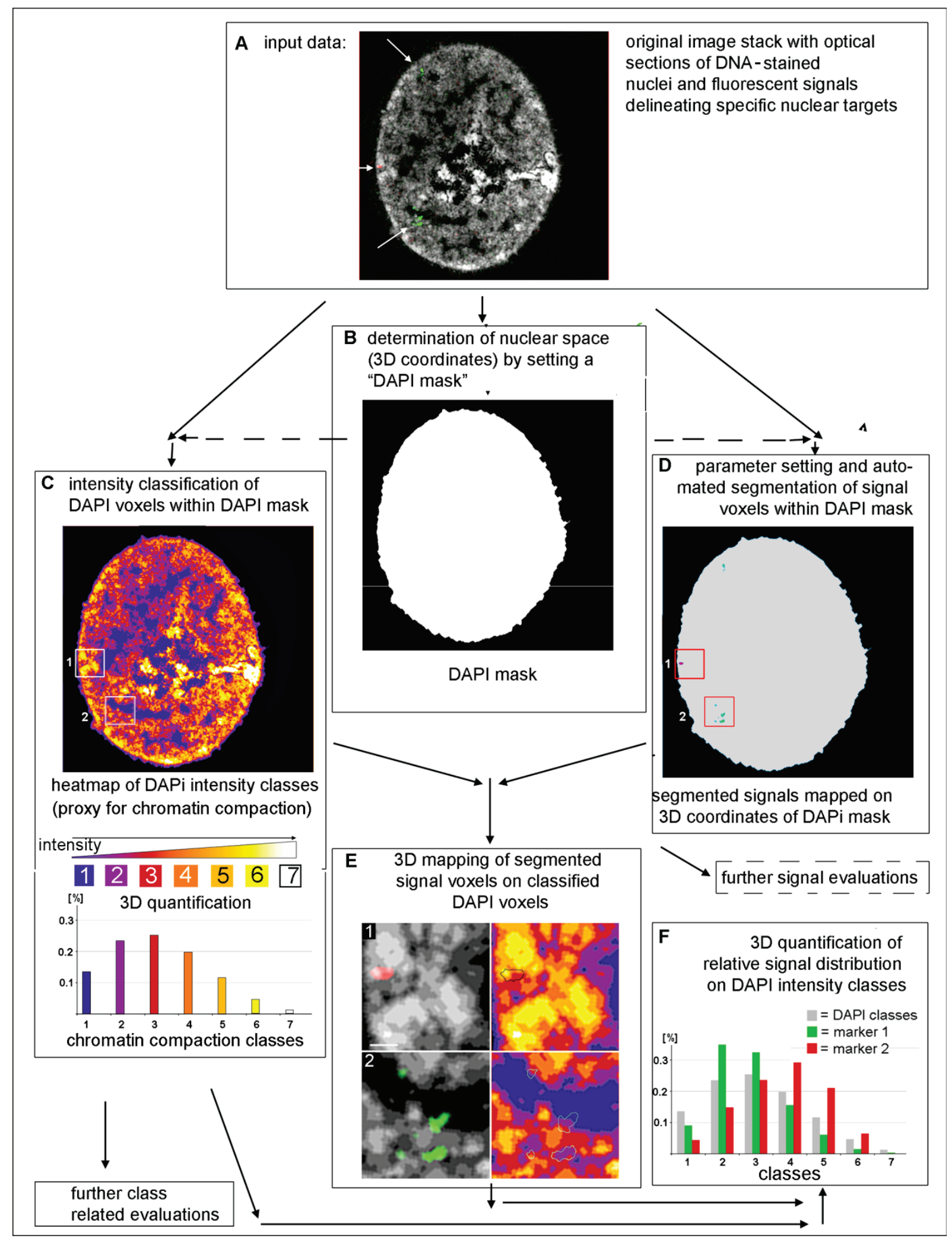

Fig. 1: Workflow for chromatin compaction classification and quantitative assignment of segmented nuclear targets on 3D chromatin compaction maps.

(A) Representative section from an original 3D-SIM image stack recorded from a fibroblast nucleus (BJ1) as starting point. DNA was counterstained with DAPI (grey). Green and red fluorescent signals (arrows) represent several differently stained DNA targets each spanning about $40 \mathrm{~kb}$. Green signals delineate DNAse I hypersensitive targets with functionally active regulatory sequences. Red signals delineate targets with inactive regulatory sequences lacking DNAse I hypersensitivity in BJ1 fibroblasts. (A full presentation of these results will be provided elsewhere: Marion Cremer, Volker J. Schmid, Felix Kraus, Yolanda Markaki, 
Andreas Maiser, Heinrich Leonhardt, Sam John, John Stamatoyannopoulos, Thomas Cremer. An initial study to demonstrate active and inactive regulatory sequences in distinct nuclear compartments by structured illumination microscopy, submitted). (B) DAPI mask defining the nuclear voxel space considered for evaluations. (C) Classification of DAPI signals into seven intensity classes visualized as color heat map (upper panel) and their relative distribution plotted as graph (lower panel). (D) Segmented fluorescent pixels used for quantitative 3D mapping on DAPI classes after defined parameter settings. These data can be used for further evaluations such as object counting and volume measurements (not considered here). (E) 3D mapping of segmented signals on DAPI intensity defined chromatin compaction classes, with outlined signals in the colored heat maps. (F) Plot of quantitative $3 D$ assignment of relative distributions for respective voxels on DAPI intensity classes.

required image quality with currently available approaches of super-resolved 3D microscopy readers are referred to $[15,18]$.

\subsection{Installation of the required software}

The software employed in our studies is implemented in the R package nucim and the accompanying package bioimagetools. Both packages and all packages on which they depend can be installed from CRAN by the commands

setRepositories (ind=c $(1,2)$ )

install.packages ("nucim")

in $\mathrm{R} .{ }^{1} \mathrm{R}$ will then present a list of CRAN mirrors around the world; choose the mirror depending on the location. For our analyses $\mathrm{R}$ version 3.3.2, nucim version 1.0.0 and bioimagetools version 1.1.0 was used. ${ }^{2}$

\subsection{Automatic Segmentation of voxels related to DAPI stained nuclear DNA}

Rationale: In a first step, we automatically segment all individual, visual sections of the DAPI stained nucleus. For this purpose, we produce masks, which outline the shape of each section and use only voxels located within the mask for further analysis.

We illustrate the analysis using an example image of a DAPI stained human fibroblast nucleus. Fig. $2 \mathrm{~A}$ shows a representative nuclear section of the DAPI-stained intensity image.

\section{Methodology}

The original image stack contains visual sections recorded above and below the nucleus. We begin by removing these irrelevant image sections. For this, we compute the mean intensity per optical section and remove sections with mean intensity below an automatically computed threshold. This threshold is computed as weighted mean of the minimum and maximum of the mean intensity per section.

In order to find the edges of the nucleus, we apply a three-dimensional variance filter, i.e., for each voxel in the image stack the variance of the voxels in a 3D-window surrounding this voxel is computed. The variance filter results in high intensities in areas with sharp features, such as the nuclear border, see Fig. 2B. An additional Gaussian smoothing filter ensures that holes in the nuclear edge are closed.

\footnotetext{
${ }^{1}$ On some operating systems, package dependencies might need additional libraries installed beforehand. For example in Debian and Ubuntu systems, these can be installed in the terminal by sudo apt install libtiff5-dev libfftw3-dev libcurl4-openssl-dev

${ }^{2}$ Additional information and development versions of both packages can be found on https://bioimaginggroup.github.io/nucim and https://bioimaginggroup.github.io/bioimagetools
} 


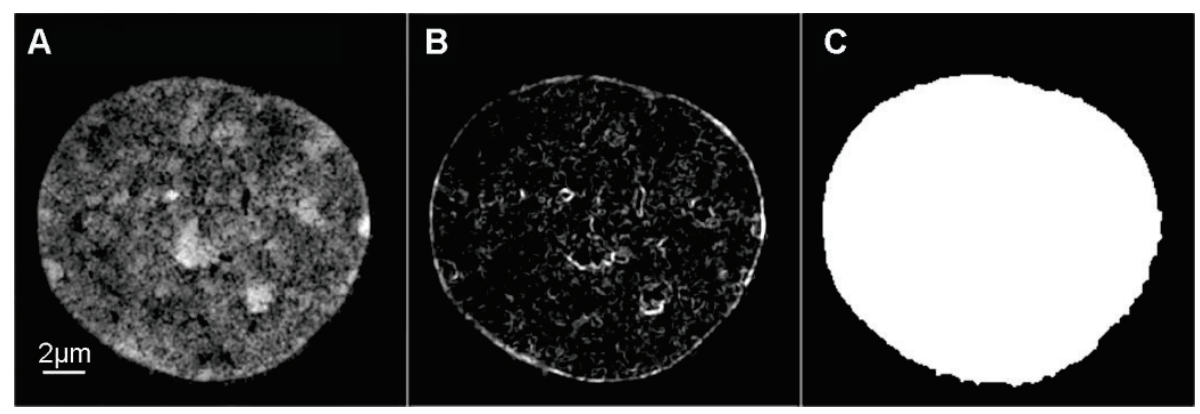

Fig. 2: Automatic masking of the nucleus using DAPI staining.

(A) Mid section of the original DAPI intensity image stack. (B) DAPI intensity image after $3 D$ variance filtering. (C) Resulting mask after Gaussian filtering, binarization and hull fill.

In order to account for possible parts of additional nuclei recorded within the same image stack, the largest object is automatically identified, while other objects are eliminated. A hull filling algorithm [19] is applied in order to close possible holes in the mask. In the following, only masked voxels are considered, see Fig. 2 C.

Size of the window and standard deviation of the Gaussian filter are determined from the voxel resolution. Next, the image is binarised by robust determination of a second threshold by simultaneously analyzing the profile in x-direction at the middle of the $y$-axis and vice versa, using different sections. In these profiles, the first and last "step“ along the profile is searched, corresponding to the edge of the nucleus.

\section{Implementation}

The automatic masking is implemented in the function dapimask() in the nucim package. Arguments of the function are the DAPI intensity image and the size of the image in microns. We load the relevant packages,

library (bioimagetools)

library (nucim)

and load the RGB TIFF file we want to analyse

img = readTIF ("someimage.tif")

The image file can also be chosen interactively

img = readTIF(file.choose())

We need the dimensions of the voxels in microns:

$\mathrm{x}=\mathrm{y}=0.0395$

$z=0.125$

Typically the third channel is blue, i.e., the DAPI intensity:

blue $=\operatorname{img}[,, 3$,

Now we can mask the nucleus:

mask = dapimask(blue, voxelsize $=c(x, y, z))$

\subsection{Quantitative 3D mapping of chromatin compaction levels based on DAPI intensity classes}

Rationale: The algorithm described below allows the quantification of DAPI intensity classes as a proxy for chromatin compaction levels and their visualization as $3 \mathrm{D}$ nuclear landscapes in single cells. DAPI related voxels are identified automatically from the DAPI channel intensities using 
Gaussian filtering and automatic threshold determination. Data sets can be used for the quantitative mapping of functionally relevant nuclear targets in relation to the various classes of chromatin compaction within individual nuclei and for comparisons between nuclei (see 2.4). Individual voxels from the DAPI channel are weighted by their intensity and segmented into seven intensity classes with equal intensity variance resulting in a signal intensity classification at the voxel level. It is possible to use more classes, when appropriate (see Discussion). Intensity class 1 comprises voxels close to background intensity representing regions that are largely void of DNA (interchromatin compartment, IC). Classes 2-7 correspond to chromatin with increasing DAPI intensities reflecting increasing levels of chromatin compaction. Class 7 represents the highest DAPI intensities and in most mammalian cell nuclei largely delineates constitutive heterochromatin such as chromocenters in mouse cell nuclei $[4,15]$.

\section{Methodology}

For chromatin density quantification, we seek to classify the voxels into seven classes based on their DAPI channel intensity. Classification is not based on fixed intensity levels or thresholds. Each voxel is assigned to a class based on the probability of this voxel of belonging to this class, computed from a stochastic model called hidden Markov random field (HMRF) model. The model uses the voxel intensity and the location of the voxel, that is, the classification of the neighbors of the voxel.

We assume that the intensity in voxel $i$ is Gaussian distributed with mean $\mu_{c}$ and variance $\sigma^{2}$, where $c$ is the class of the voxel. This leads to a mixture of Gaussians for the distribution of all voxel intensities, as can be seen in Fig. 3. The classes are latent, that is, they cannot be observed directly. Therefore, we use the so-called Potts model for the classes per voxel as hidden layer in the HMRF. That is, we assume that neighboring voxels, i.e., voxels sharing a border, are more likely to belong to the same class. This assumption makes the algorithm more robust, although the differences in the results are usually rather subtle. The Gibbs energy (or negative log probability density) of the Potts model is defined as

$$
\beta \sum_{i \sim j} I\left\{c_{i} \neq c_{j}\right\}
$$

where $I$ is the indicator function, which is one if, in this case, $c_{i}$ is equal to $c_{j}$ and zero otherwise, and $i \sim j$ means voxel $i$ is neighbor of voxel $j$. The parameter $\beta$, known as "inverse temperature", determines how much neighboring voxels influence each other.

Combining the mixture of Gaussians with the Potts model in the HMRF can be done in a Bayesian framework. Here, the Potts model serves as prior distribution for the unknown classes. We use uninformative prior distributions for the parameters $\mu_{c}$ (expected values per class) and $\sigma^{2}$ (variance). Before classification, the DAPI intensity image is internally transformed into a 16bit integer array. Inference is done using a Conditional Expectation Maximization (CEM) algorithm [10]. For the CEM, we start with a random classification. Given the classification the expected value $\mu_{c}$ can easily be computed as mean of the intensities of voxels belonging to class $c$. Then the variance $\sigma^{2}$ is estimated. The algorithm sorts the classes by $\mu_{c}$ and, in case of empty classes, splits up the class with most voxels. Voxels are assigned to the class with the highest probability, which depends on their intensity and the class of the neighboring voxels. The algorithm iterates and terminates when an iteration does 


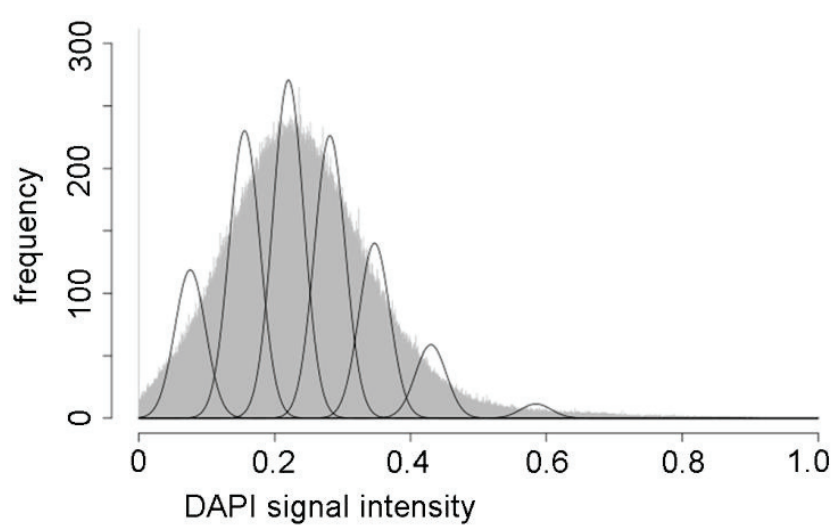

Fig. 3: Histogram and Gaussian mixture distribution

Histogram of the DAPI intensity of the example image in grey, where only the voxels in the mask where used. In black, probability density functions of the seven Gaussians found by the Conditional Expectation Maximization (CEM) algorithm. Using the Gaussian mixture, the voxels are assigned by their probability of belonging to one of the Gaussians, which not only depends on the Gaussians, but also on the classes of neighboring voxels.

not lead to a change in voxel classification. The CEM algorithm results in the configuration with the maximum a posterior (MAP) probability.

The hidden Markov random field model used here allows classifying the DAPI image without the need to specify arbitrary thresholds, but based on the actual intensity distribution of the image. Classifications of a very heterogeneous group of images therefore might not be comparable, but the results show that the classifications in a homogenous group of images can very well be compared.

\section{Implementation}

Classification of DAPI intensity images is implemented in the classify() function in the nucim package. Arguments of the function are the DAPI intensity image, the mask, the inverse temperature $\beta$ and a scaling parameter for the inverse temperature between optical sections. The DAPI intensity should be stored at least in 16bit. Default for the inverse temperature is $\beta=0.1$. The scaling parameter for the influence of voxels between optical slices is computed as size of voxels in X-/Y-direction divided by size of voxels in Z-direction.

classes $=$ classify (blue, mask, 7, beta=0.1, $z=x / z$ )

In the resulting object each voxel has assigned the number of the chromatin compaction class it belongs to or zero if the voxel is outside the mask. From this, we can count the number of voxels per class and plot this, see Fig. 4A,

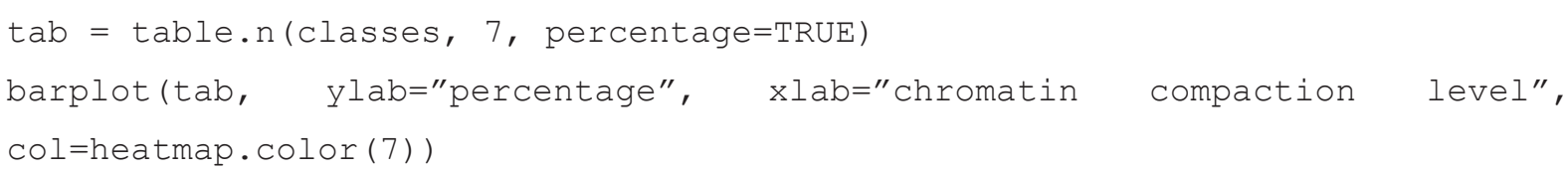

The function heatmap.color (7) provides an adequate color palette for seven levels. This can also be used for plotting a map of the chromatin compaction levels for an optical section, see Fig. 4B:

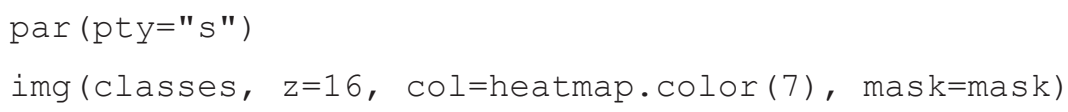




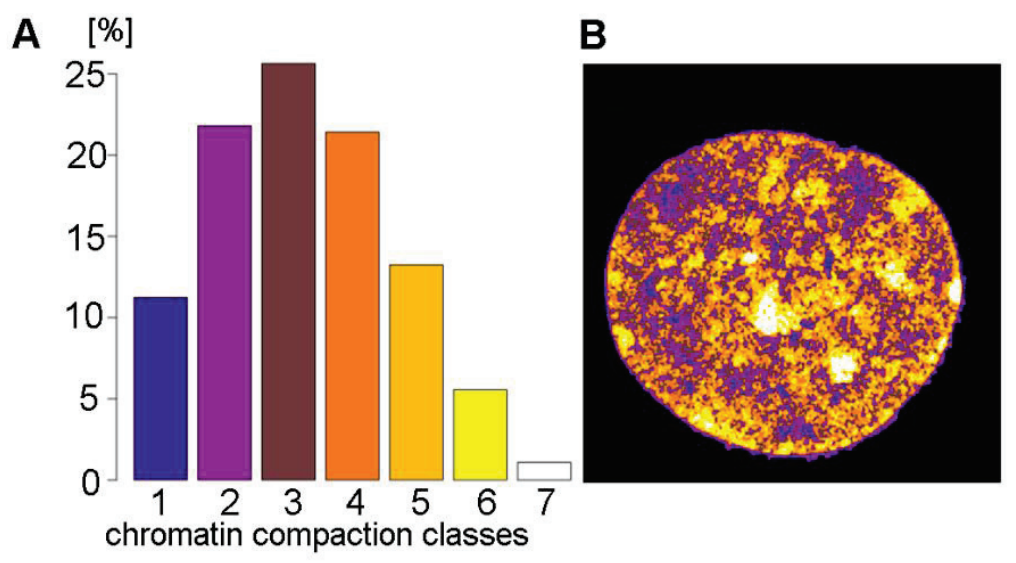

Fig. 4: Mapping of chromatin compaction levels.

(A) Barplot of the distribution of chromatin compaction levels based on 7 DAPI intensity classes. (B) Color heat map of chromatin compaction levels in one optical section (same optical section as in Fig. 2) matches the colors of the chromatin compaction levels in the barplot.

The classified image can also be saved for processing in other software tools, e.g. ImageJ.

writeTIF(classes, "classes.tif")

The classified image can be reloaded into $\mathrm{R}$ using

classes = readclassTIF("classes.tif")

\subsection{Measurements of nearest neighbor voxels and absolute distances between DAPI intensity classes}

Rationale: Class assignment of nearest neighbor voxels in 3D for a given DAPI intensity class substantiates the visual appearance of nuclear landscapes in the context of a compaction shaped pattern formation. For example, chromatin domain clusters (CDCs) are a typical feature of eukaryotic cell nuclei with a shell-like organization, resembling an onion skin. A CDC is composed of an interior compact chromatin core (intensity classes 5-7) and a peripheral decondensed layer chromatin layer (classes 2-3). Such a pattern will correspond to most nearest neighbor voxels belonging to the same or next higher or lower intensity class, and only rare voxels to more remote classes. Minimal absolute distances between voxels, which belong to a given intensity class, to the nearest voxel of any other class can be estimated as a proxy to assess the minimal covered distance of a given nuclear target switching between different intensity classes, e.g. regulatory sequences in response to transcriptional stimulation. We describe an approach for the measurement of mean minimal distances between respective voxels described by their xyz centroid coordinates.

\section{Methodology}

For the measurements of distances between chromatin compaction classes, for each voxel the nearest neighbor for each level is searched in 3D. The search is performed iteratively starting in a small, than increasing 3D neighborhood. As this is done for each voxel in the mask, the computation is rather time consuming, but can easily be performed in parallel, if more than one CPU core is available (as in most modern computers). 

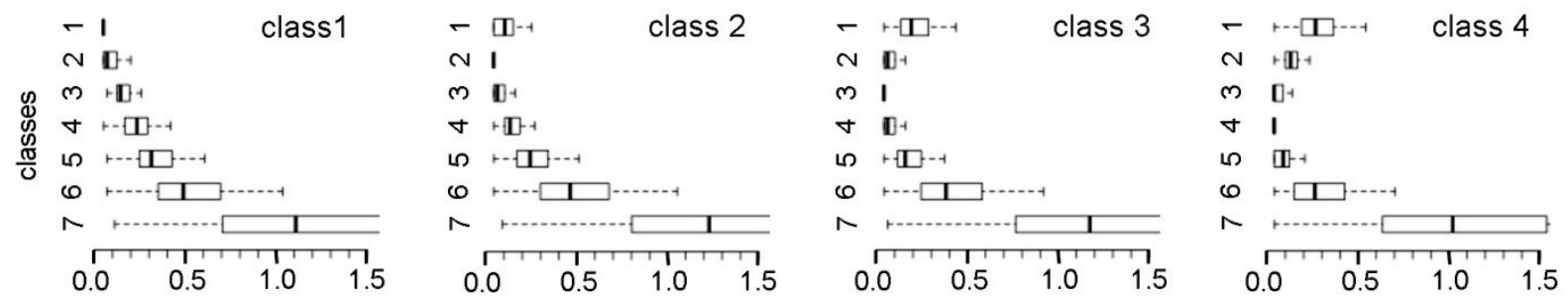

distance to nearest neighbour $[\mu \mathrm{m}]$
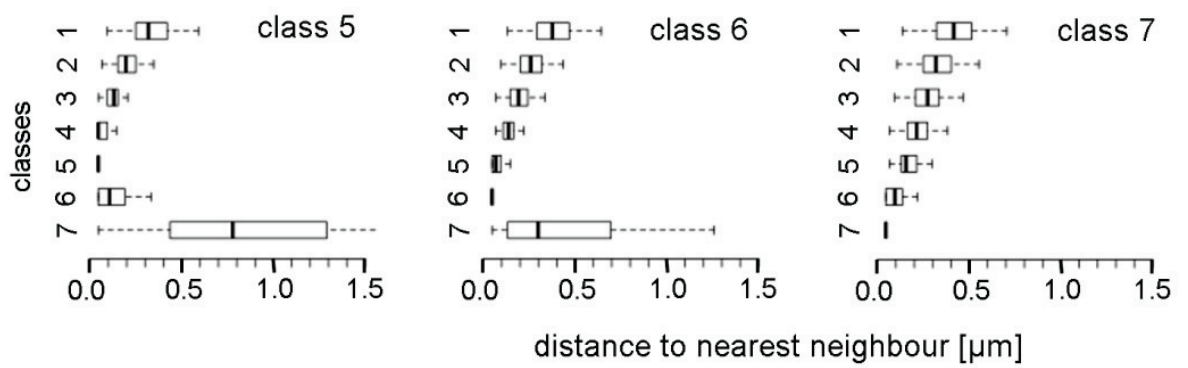

Fig. 5: Boxplots representation of distances between chromatin compaction classes.

For each chromatin compaction class, a boxplot of the nearest neighbor distances between all voxels attributed to a given class and the nearest neighbor voxel attributed to other classes is shown. The boxplots show median, $25 \%$ and $75 \%$ quantile; the whiskers show the range of values. Most nearest neighbors belong to the same intensity class, a smaller fraction to the next higher or lower class and only rare voxels to remote classes. This result supports a shell-like structure of CDCs composed from layers of different chromatin compaction levels.

The distances are summarized and visualized for each class. For example, boxplots can be used, see Fig. 5. Alternatively, the minimum or, in order to be robust for outliers, a very small quantile of the absolute distance to the next neighbor can be visualized, see Fig. 6 .

Distances are computed as distances between centroids of voxels. Note that these computed distances depend on the image resolution, i.e., the real size of voxels, and can be smaller than the actual optical resolution of the imaging system.

\section{Implementation}

For the computation of next neighbor distances of compaction levels the nearestClassDistances () function in the bioimagetools package can be used. Arguments of the functions are the image of chromatin compaction classes, the actual size of the voxels, the number of classes and the number of cores for parallel computing:

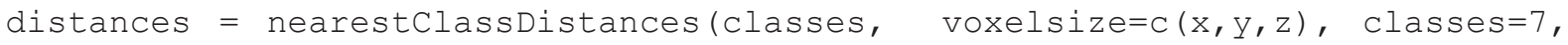
cores $=4$ )

We highly recommend using parallel computing, which is possible on UNIX/Linux systems and macOS computers. The number of cores used in parallel is controlled by the option cores. The distances can be plotted using plotNearestClassDistances (), e.g. for Fig. 5:

plotNearestClassDistances (distances, method="quantile")

and for Fig 6:

plotNearestClassDistances (distances, method="boxplot") 

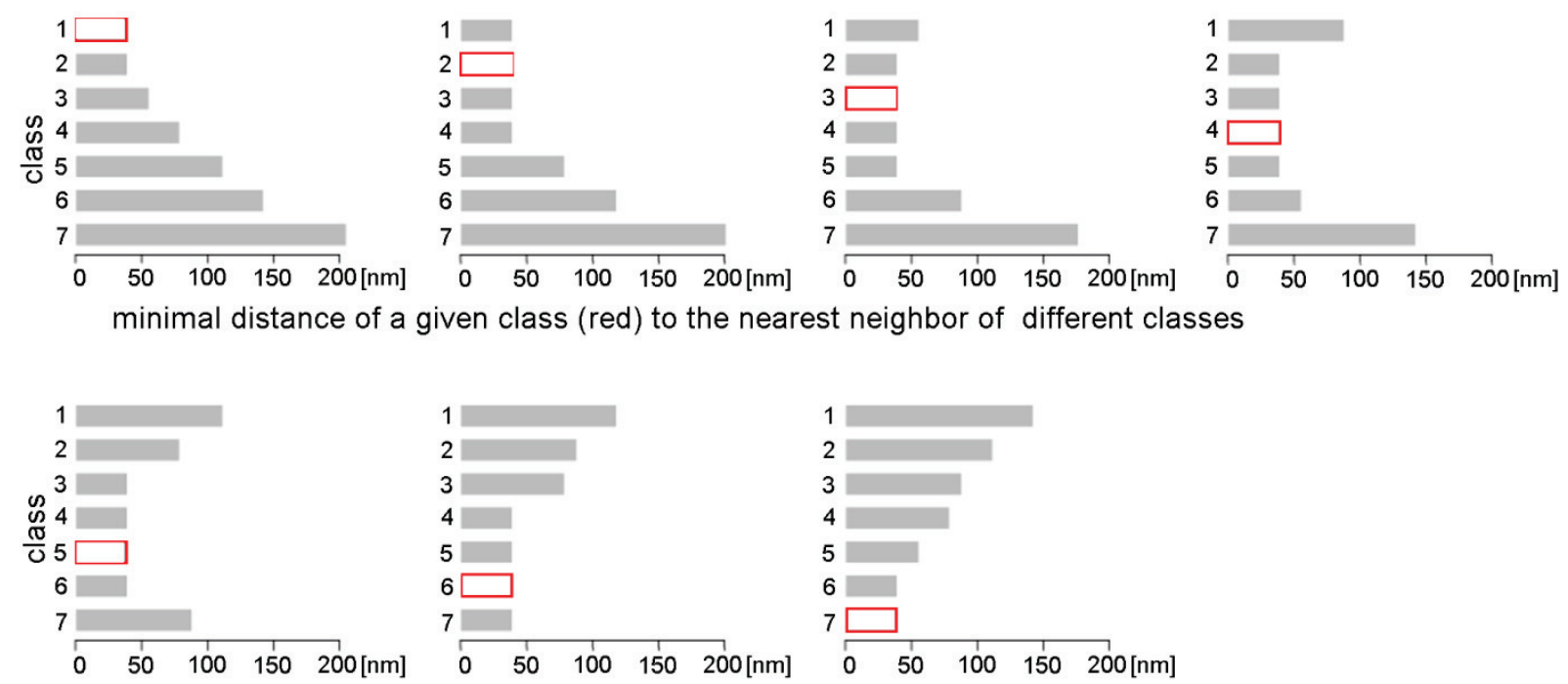

minimal distance of a given class (red) to the nearest neighbor of different classes

Fig. 6. Minimum of distances between chromatin compaction classes.

For each chromatin compaction class (marked red), all distances between voxels belonging to this class and voxels belonging to other classes were computed. From all computed distances the minimal nearest neighbor distance between the red marked class and other classes was identified and shown here. This visualizes a lower bound of the distances between chromatin compaction classes. The minimal distance between classes 1 (largely representing the interchromatin compartment) and class 7 (the most compacted chromatin compartment) is roughly $200 \mathrm{~nm}$, i.e. at the Abbe limit of conventional light microscopic resolution.

\subsection{Quantitative 3D mapping of specific DNA sequences and nuclear proteins relative to DAPI intensity defined chromatin compaction maps}

Rationale: This section describes the quantitative 3D mapping of specific DNA sequences or nuclear proteins in relation to DAPI intensity classes. A functional link between nuclear landscapes with distinct differences of chromatin compaction and biologically relevant markers was previously established by quantitatively mapping the relative spatial distribution of RNA-polymerase II, nuclear bodies and histone modifications representing markers for either transcriptional competent chromatin or a silent chromatin state to seven DAPI intensity classes [4]. The compacted core of CDCs (classes 5-7) constitutes the inactive nuclear compartment (INC). The active nuclear compartment (ANC) is formed by the interchromatin compartment (IC; class 1) together with the decondensed peripheral layer of CDCs (classes 2-3/4), called perichromatin region (PR). The IC starts/ends at nuclear pores, pervades the nuclear interior as a channel system, which is co-aligned with the higher order chromatin network built up from CDCs. IC-channels expand occasionally into wider lacunas, which carry splicing speckles and other nuclear bodies. These structures serve factors or aggregates for nuclear functions carried out within the PR. Notably, the displacement of chromatin by such structures does not suffice to explain the very low DAPI intensity recorded within IC-channels in general.

In addition to the mapping of functionally relevant proteins on the higher order chromatin landscape, specific DNA sequences, such as coding or transcription regulatory sequences (TREs) as well as RNA sequences [16] can be assigned to different DAPI intensity classes, representing the ANC or the INC. In case of 3D mapping of small single copy nuclear targets we provide an optional additional tool 
for an unbiased automatic discrimination between small background dots and true signals by differential labeling of a contiguous target sequence or protein complex and setting of a maximal distance between them.

\section{Methodology}

We recommend a combination of two approaches in order to map additional markers to different chromatin compaction levels: First, a manual or semi-automatic threshold can be used in order to suppress noise and to identify marked proteins/DNA/RNA targets. Second, marker voxels are weighted with their intensity, with clear marker signals having large impact and low intensity noise having very small impact.

In special cases specific segmentation algorithms for markers can be applied, for example a shape criterion can be introduced in case that the signal of a marked protein aggregate is known to be roughly circular. Such segmentation can also be done using commercial software like Volocity (Perkin Elmer, Waltham, MA, USA), Imaris (Imaris scientific 3D/4D image processing \& analysis software. Bitplane), Cell Profiler [20] or ImageJ plugins like 3D Object Counter and JACoP [21]. However, this high additional effort of object segmentation is often not necessary, as small amounts of noise typically do not have a high influence on the end results.

In case of small single copy DNA sequences targeted by respective DNA-probes, the unequivocal distinction of a true hybridization signal from a dotted unspecific signal can become a challenge for correct signal assignment in 3D FISH experiments. In such cases a few unspecific signals included in an evaluation can considerably influence a quantitative analysis. Using two or more contiguous, differentially labeled probes for such sites helps to overcome this problem. We therefore implemented 3D distance measurements between the 3D centroid positions of a given signal to its nearest differentially colored signal (Fig. 7A).

In case of small contiguous differently labeled target sequences, we first use adaptive thresholding to find potential signals [19]. In 3D connected voxels above threshold are identified as signal objects and total intensity and centroid are computed for these. Signal objects with low total intensity are disregarded. Then, distances of the centroids of the potential signals of different labeling are computed in order to identify contiguous target sequences and only signals below a defined centroid distance (e.g. $\leq 500 \mathrm{~nm}$ ) are accepted as true hybridization events.

After thresholding, we weight each voxel in a chromatin compaction class by its actual signal intensity. This way, clear signals will have high weight in our analysis and signals with low intensity, which cannot easily be discriminated from noise, will have low impact on the end result.

The number of voxels per chromatin compaction class can then be visually compared with the weighted number of marker signal voxels per chromatin compaction class, for example by plotting the relative enrichment (overrepresentation) or depletion (underrepresentation) of marker signals in each chromatin compaction class. Please note that enrichment and depletion can either be presented in relative percentages, that is, an enrichment of $100 \%$ means a doubling of the proportion of this class (as seen in Fig. 9). Or it can be visualized as changes of percentage points, that is, a change from $5 \%$ to $10 \%$ would result in an enrichment of 5 percentage points [16]. Which of the two possibilities may be preferred, depends on which aspect of the relative enrichment or depletion should be emphasized. 
The differences in the distribution of chromatin compaction class voxel in general and of the chromatin compaction voxel with a specific signal, but also for the distribution of two different marker signals can be statistically tested. A Chi-squared test for contingency tables is appropriate, as it does not depend on a distribution assumption. Alternatively, a Wilcoxon rank-sum test, also known as Mann-Whitney Utest, can be used for testing shifts of the mean of the distribution.

\section{Implementation}

First, we separate the red and green channel from the RGB image

red $=\operatorname{img}[,, 1$,

green $=\operatorname{img}[, 2$,

The function colors.in.classes() provides the methods for computing the chromatin class distributions in general and per marker signal. Additionally it provides plots and statistical tests. We start with a threshold based approach using the previously derived chromatin compaction class image and the nucleus mask. Here, the threshold is fixed to 0.1 for both markers. Plotting is enabled with the argument $\mathrm{plot=TRUE,} \operatorname{col} 1$ and $\operatorname{col} 2$ give the color of the bars corresponding to the markers. $\mathrm{ylim}$ defines the limits of the $y$-axis, this is fixed here for comparison between figures. The Wilcoxon ranksum test is computed using the argument test="Wilcoxon":

cic1 = colors.in.classes(classes, red, green, mask, $\mathrm{N}=7$, type = "thresh", plot = TRUE, $\operatorname{col} 1=$ "red", $\operatorname{col} 2=$ "green", thresh1 = 0.1, thresh2 = 0.1 , test $=$ "Wilcoxon", ylim $=\mathrm{c}(0, .8)$, xlab = "chromatin compaction levels", $\mathrm{ylab}=$ "percentage")

The result is depicted in Fig. 7B. We see a clear enrichment of the labeled sequences in chromatin compaction level 2. All results are stored in the object $\mathrm{cic} 1$ for subsequent analysis. The results of the Wilcoxon rank-sum tests are:

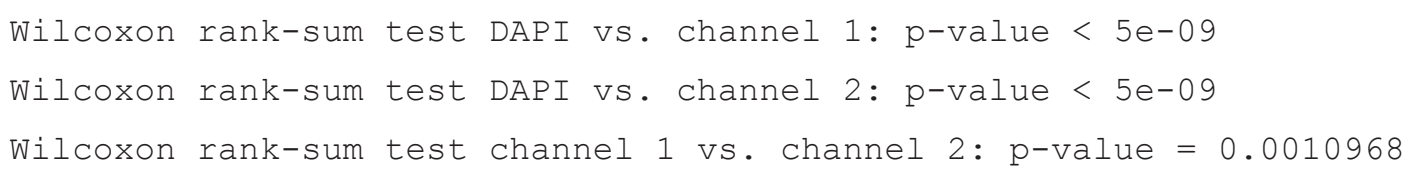

Here, the general distribution of chromatin compaction classes and the distribution of both marker signals in the chromatin compaction classes is statistically different. The distribution of both marker signals is also different.

The argument type="intensity" will compute distributions and tests based on the intensityweighted method. Again, we use a threshold of 0.1 for background suppression; signal above the threshold will be weighted by its intensity:

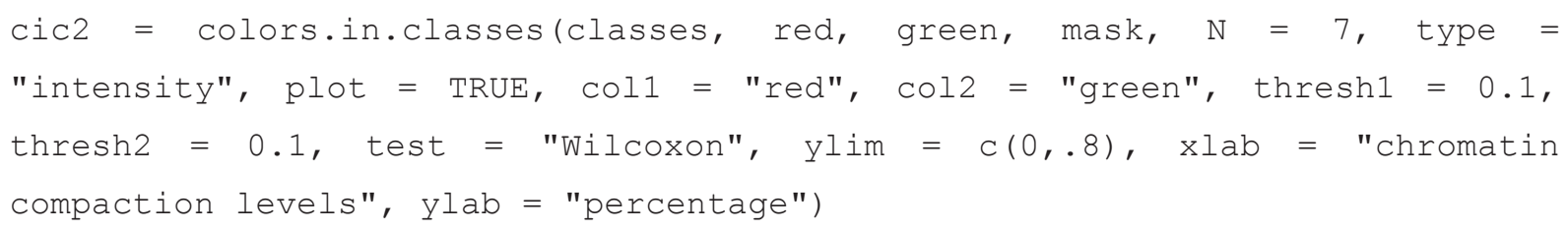

The resulting graphic can be found in Fig. 7C. The enrichment in level 2 is even more distinct here. The results of the Wilcoxon rank-sum tests are similar as above, but the distributions of both markers is not statistically on a $5 \%$ level - actually these are contiguous differently labeled target sequences:

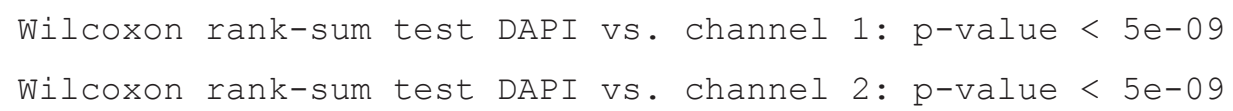



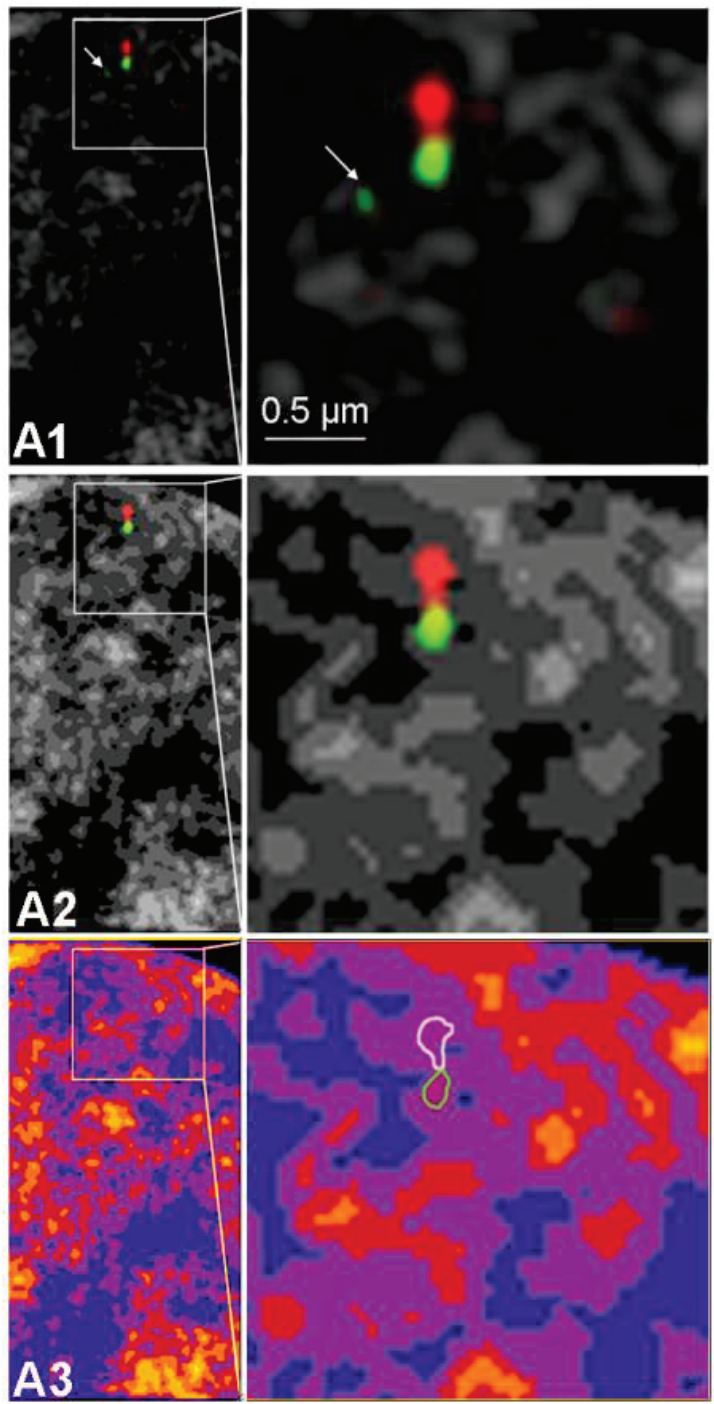
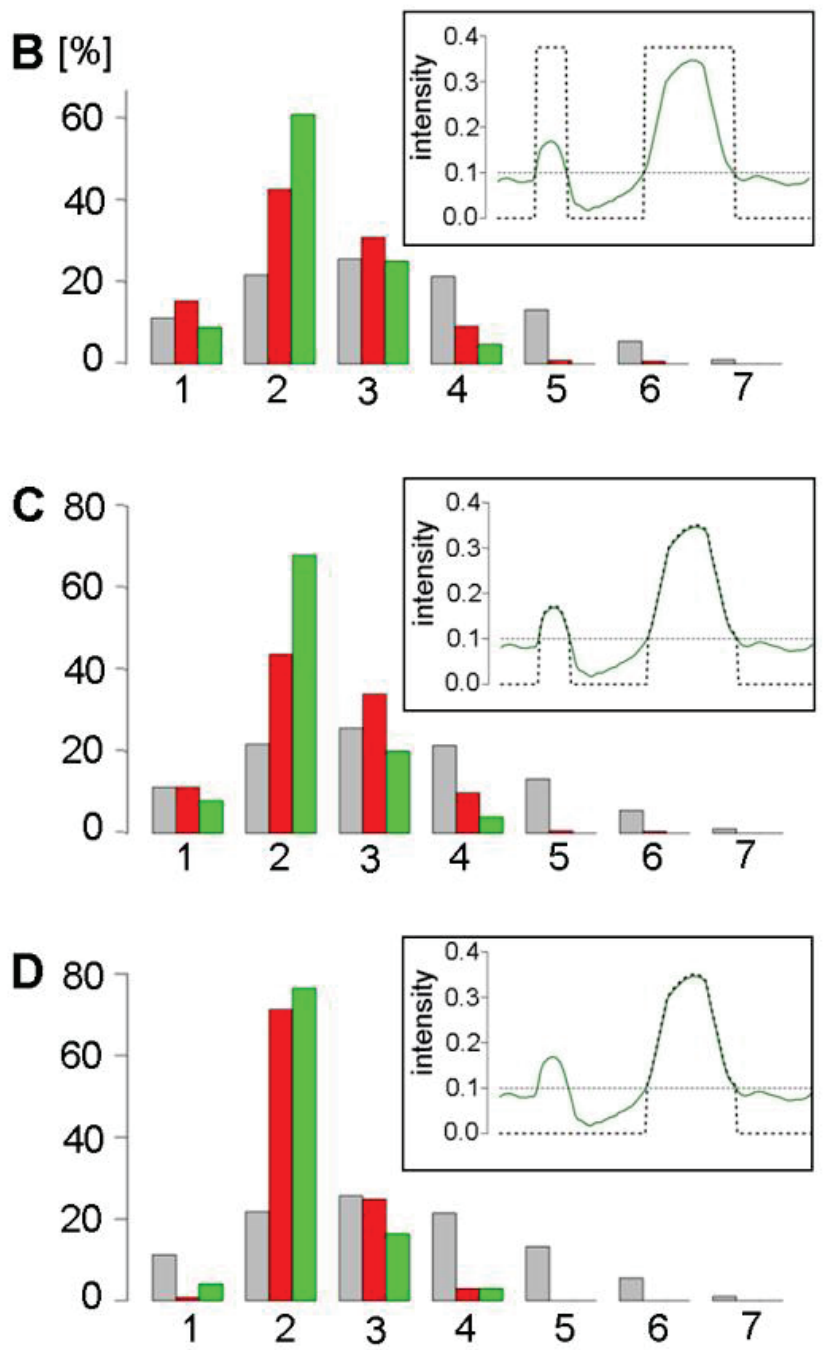

chromatin compaction classes

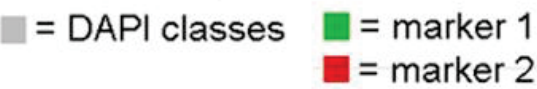

Fig. 7. 3D mapping of two contiguous DNA probes relative to chromatin compaction classes

(A) Partial section shows adjacent green and red 6 kbp DNA targets from chromosome 2, which are also adjacent along the linear DNA level. The section was taken from a 3D image stack recorded with SIM from a DAPI stained, diploid fibroblast nucleus (shown in gray). Targets were detected by 3D FISH with differentially labeled DNA probes. The green target represents a DNA hypersensitive site in BJ cell nuclei. Further details will be published elsewhere (A full presentation of these results will be provided elsewhere). (A1) Original image. (A2) Same image after DAPI intensity classification. As a criterion for dotted background, any small fluorescent dot with a distance $>0.5 \mu \mathrm{m}$ from the nearest signal centroid of a clearly identifiable target was attributed to background and eliminated from further consideration after signal segmentation with appropriate parameter settings. The additional green fluorescent background dot (indicated by arrow in A1) presents an example. (A3) The same image presented as a color heat map of chromatin compaction classes (compare Fig. 4) with schematically outlined targets. (BD) Barplots of voxel percentages for chromatin compaction levels (grey), and percentages of red and green markers for each chromatin compaction level. The barplots show an enrichment of both signals in class 2. Inlay: Visualization of weighting of markers signal intensity for a profile: Signal intensity in green, threshold as dotted line, dashed line as weight used for the respective voxel. (B) Result using an arbitrary threshold. Inlay shows that all voxels above the threshold have equal weight. (C) Result using thresholding and weighting proportional to voxel signal intensity. Inlay shows that voxel with marker signal intensity below threshold are weighted with zero, all voxel above threshold are weighted with their marker signal intensity. (D) Result using contiguous differently labeled target sequences. Inlay shows that green spot voxels with larger distances to nearest red spot (see A1) are weighted to zero. 
Wilcoxon rank-sum test channel $1 \mathrm{vs}$. channel 2: p-value $=0.06307286$

We make use of the fact that we have contiguous differently labeled target sequences by finding spots using the function spots. combined ():

spots $=$ spots.combined(red $=$ red, green $=$ green, mask = mask, voxelsize = $\mathrm{C}(\mathrm{x}, \mathrm{y}, \mathrm{z})$, full.voxel = FALSE, thresh.offset $=0.05)$

The resulting spots object includes masked images of the red and green spots, which we can use in colors.in.classes ():

cic3 = colors.in.classes(classes, spots\$red, spots\$green, mask, $N=7$, type = "intensity", plot = TRUE, $\operatorname{coll}=$ "red", $\operatorname{col} 2=$ "green", test = "Wilcoxon", ylim = c(0,.8), xlab = "chromatin compaction levels", ylab= "percentage")

The results of the Wilcoxon rank-sum tests are as follows:

Wilcoxon rank-sum test DAPI vs. channel 1: p-value $=6.244 \mathrm{e}-05$

Wilcoxon rank-sum test DAPI vs. channel 2: p-value $=0.01004648$

Wilcoxon rank-sum test channel $1 \mathrm{vs}$. channel 2: p-value $=0.902997$

The resulting barplot is pictured in Fig. 7D. The enrichment of the differently labeled target sequences is even more pronounced here. The Wilcoxon test shows no difference in the distribution of the signals, which should be expected as we only use contiguous signals. The power of the statistical tests is a little bit smaller, as we use less signals here. However, the results are still very clear in this case.

\subsection{Analysis of multiple cell nuclei}

Rationale: Quantitative image analyses of nuclei from multiple cells are mandatory for the analysis of intercellular variations. It should be emphasized, however, that such comparisons must be performed with caution to avoid an over-interpretation of apparent cell-to-cell differences. Cell cycle related variances between nuclei, an exceptional pattern formation in the nucleus, noise or artifacts in the image at hand can lead to misleading conclusions. It is is crucial to acquire several images from nuclei on the same slide and to analyze them together. As a consequence of systematic, but unrecognized differences in the application of complex protocols, comparisons of nuclei from different slides can be more problematic than comparisons of nuclei from cells present on the same slide, in particular, when slides stem from independent experiments.

\section{Implementation}

The nucim package provides function to process all images in a folder sequentially or in parallel and store the results in appropriately named folders. For example, let us assume we have a couple of RGB tiff stacks in the folder "rgb" in the folder " / my_study". The code

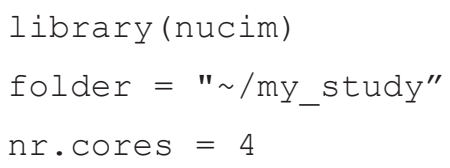


splitchannels.folder(folder, rgb.folder="rgb", cores=nr.cores)

will read in all tiff stacks in folder " / my_study" and for each stack writes out the red, green and blue channel respectively in folders "red", "green" and "blue". The files of the tiff stacks have the same name as the original file in the folder "rgb". If $n r$. cores is greater than 1 , this is done using parallel computation.

nucim provides functions to process all steps in section 2.2 to 2.5 for all images in a folder. For example,

dapimask.folder(folder, voxelsize $=c(0.0395,0.0395,0.125)$, cores = nr. cores)

computes masks for each tiff stack based on the blue channels in folder "blue" and writes them in a folder "dapimask". The voxelsize argument gives the size of a voxel in microns, as above. Although the algorithms are quite robust, intermediate results for this and all following steps should always be checked for artifacts.

classify.folder(folder, 7, cores = nr.cores)

computes the chromatin compaction levels for each tiff stack. The resulting tiff stacks with the classification are in a folder called "class7" (with 7 classes, "class11" for 11 classes and so on) and the counts of voxels per class are in a folder "class $7-n$ ". The counts and percentages are saved in text files with the same name as the original tiff stacks, but with the additional ending ". txt". These files are used for example to plot the mean percentage of voxels per chromatin compaction level along with the range of percentages:

plot_classify.folder(folder, 7, col = heatmap.color(7), cores $=$ nr.cores)

Fig. 8A shows an example: the mean distribution of voxels belonging to chromatin compaction classes over a series of cell nuclei $(n=20)$ along with error bars which visualize the standard deviation of the percentage per class over nuclei.

3D mapping of marked proteins relative to DAPI intensity classes can be done by

colors.in.classes.folder(folder, "green", color2="red", thresh1 = 0.05, thresh2 $=0.05$, cores $=$ nr.cores)

plot_colors.in.classes.folder(folder, "green", "red")

t_colors.in.classes.folder(folder, test = "Wilcoxon ")

or in case of contiguous differently labeled target sequences by

spots.combined.folder(folder, voxelsize=c(0.0395, 0.0395, 0.125), cores = nr.cores, thresh.offset $=0.05$ )

colors.in.classes.folder(folder, "markers_green", color2 = "markers_red", coll = "green", $\operatorname{col} 2=$ "red", cores = nr.cores, type = "i")

plot_colors.in.classes.folder(folder, "green", "red")

The resulting plots are shown in Fig. 8B.

The Wilcoxon test can also be done using information from all cells:

t_colors.in.classes.folder(folder, test = "Wilcoxon") 

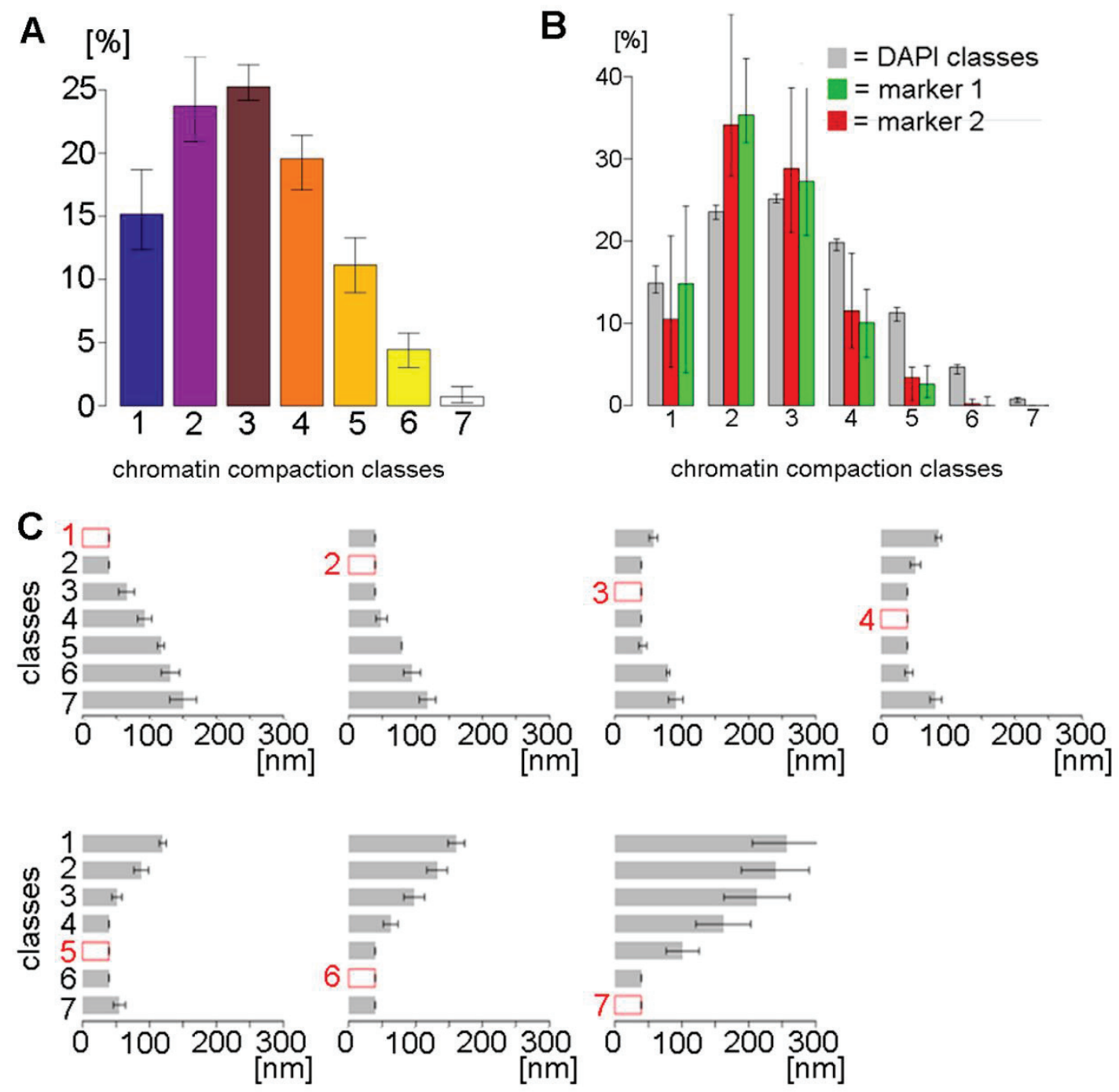

minimal distance of a given class (red) to nearest neighbor of different classes

Fig. 8 Results for the analysis of multiple cell nuclei

A complete 3D image analysis of 3D SIM image stacks recorded from 20 BJ1 cell nuclei. Same experimental setup as explained in Fig. 7. (A) Barplot of mean distribution of voxels in chromatin compaction classes (similar to Fig. 4A) with standard deviation error bars. (C) $3 D$ mapping of marked DNA-targets relative to these classes. (C) Minimal distances between chromatin compaction classes were computed for the set of 20 nuclear image stacks (compare Fig. 6) and are presented with standard deviation error bars.

Here this gives the result:

Wilcoxon rank-sum test DAPI vs. channel 1: p-value $=3 e-07$
Wilcoxon rank-sum test DAPI vs. channel 2: p-value $=1.536 \mathrm{e}-05$
Wilcoxon rank-sum test channel $1 \mathrm{vs}$. channel 2: p-value $=0.3222579$

Likewise, the computation of the minimal distances of a voxel from a given class to the nearest neighbor from another class can be done on all tiff stacks from the entire set of nuclei kept in a folder. The results are saved in a folder "distances" and summarizing plots can be generated by 
A

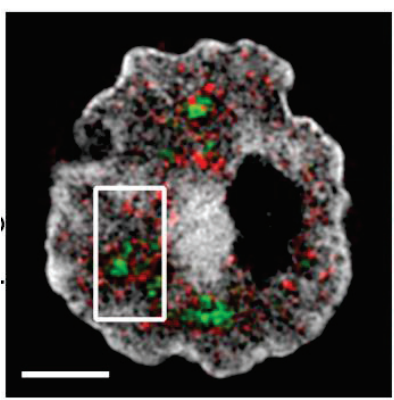

DAPI SC35 RNA Pol II

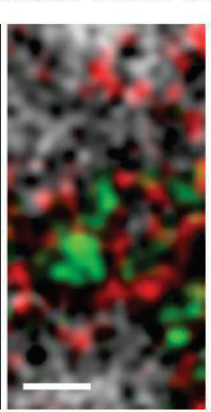

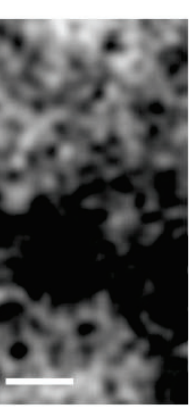

DAPI

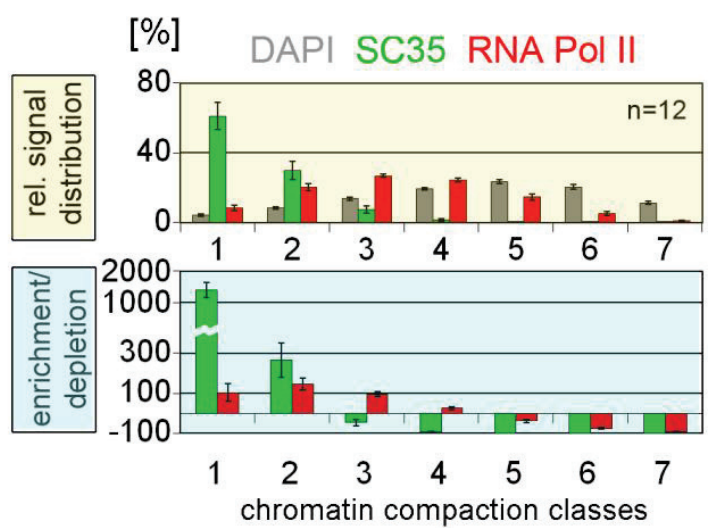

B

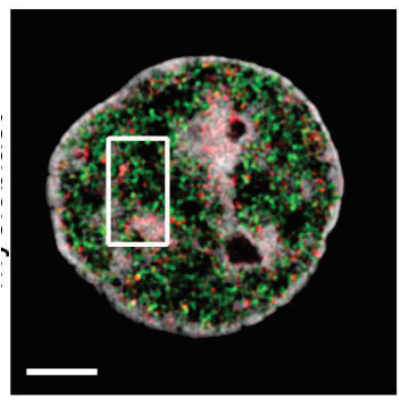

hematopoietic precursor cell

DAPI H3K4me3 H3K9me3

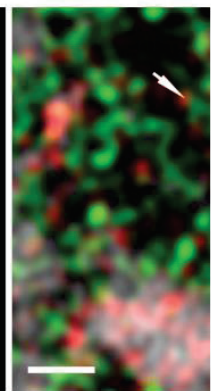

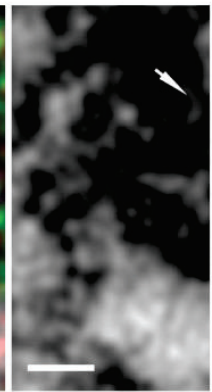

DAPI
[\%]
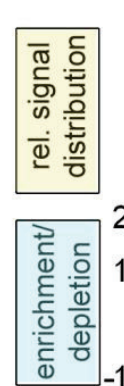

DAPI H3K4me3 H3K9me3
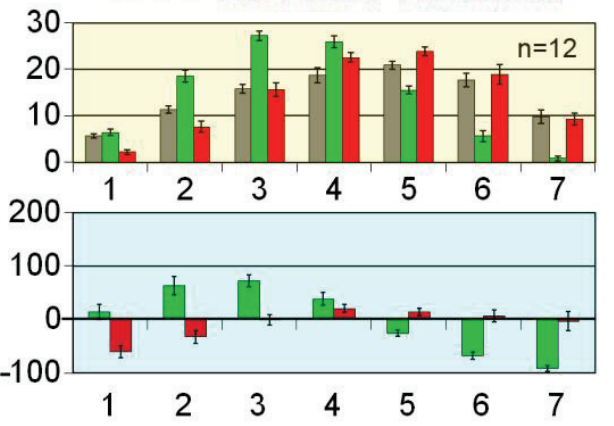

chromatin compaction classes

Fig. 9: Quantitative assignment of markers reflecting transcriptional activity or repression on $3 D$ chromatin compaction maps in a sample of hematopoietic human cell nuclei (modified from [14]).

(A) RNA Pol II with Serine 5 phosphorylation (Ser5P) is involved in the initiation of transcription, whereas SC35 accumulates in splicing speckles, which serve ongoing transcription with factors for co-transcriptional splicing. Left: light optical mid-section from a $3 D$-SIM image stack recorded from a hematopoietic stem cell nucleus and representative inset magnification delineating DAPI stained DNA (gray), immuno-stained SC35 (green) and RNA Pol II (Ser5P) (red). Right: Quantitative assessment of signals on DNA intensity classes obtained from 12 nuclei confirms a distinct localization: SC35 is found as an integral part of splicing speckles almost exclusively in the IC compartment, while RNA Pol II shows a preferential localization at decondensed chromatin sites or at the surface of compacted chromatin domain clusters. Scale bars: $2 \mu \mathrm{m}$; insets $0.5 \mu \mathrm{m}$. Graphs highlighted with yellow background: relative signal distribution of SC35 and RNA Pol II within the respective DAPI/ chromatin compaction classes (compare Fig. 4). Graphs highlighted with light-blue background: quantified levels of relative enrichment (positive values) or depletion (negative values) of SC35 and RNA Pol Il signals relative to the DAPI signals confirm the massive enrichment of SC35 signals in class 1 reflecting the IC compartment. p<0.001 for DAPI vs. SC35 and RNA Pol II, and for SC35 vs. RNA Pol II. (B) H3K4me3, a histone marker for transcriptional competence and H3K9me3, a global histone marker for transcriptionally repressed (hetero)chromatin. Left: 3D-SIM light optical mid-section from a 3D SIM image stack recorded from a hematopoietic precursor cell nucleus and representative inset magnification delineating DAPI stained DNA (grey), immuno-stained H3K4me3 (green) and H3K9me3 (red). H3K4me3 marks decondensed chromatin sites. H3K9me3 marks highly compacted chromatin clusters but is also found at decondensed sites (arrows). Scale bars: $2 \mu \mathrm{m}$; insets $0.5 \mu \mathrm{m}$. Right: This observation was confirmed by quantitative assessment of signals recorded in 12 nuclei on chromatin compaction classes (compare Fig. 4). Graphs highlighted with yellow background: relative signal distribution of H3K4me3 and H3K9me3 within respective DAPI defined chromatin compaction classes. p<0.001 for DAPI vs. H3K4me3 and H3K4me3 vs. H3K9me3. Graphs highlighted with light-blue background: quantified levels of relative enrichment (positive values) or depletion (negative values) of H3K4me3 and H3K9me3 signals relative to DAPI signals reveal an enrichment of H3K4me3 in low intensity classes and depletion of H3K9me3 signals in classes 1 and 2. $n=$ number of analyzed nuclei; error bars = standard deviation. 
nearestClassDistances.folder(folder, cores = nr.cores)

plot_nearestclassDistances.folder(folder, cores = nr.cores)

Fig. $8 \mathrm{C}$ depicts the result.

Fig. 9 exemplifies the comparative topology of markers reflecting either transcriptional activity/competence or transcriptionally silenced chromatin in samples of hematopoietic cell nuclei.

\section{DISCUSSION}

\section{DNA and chromatin stains}

In our experiments DAPI staining to saturation was used as a proxy for measurements of $3 \mathrm{D}$ chromatin compaction at the resolution level of 3D-SIM [16]. This fluorescent dye served our needs best because its high photostability, DNA specificity and spectral properties meet the demands of the 3D-SIM system configuration available at the LMU Biocenter. The binding preference of DAPI to ATrich DNA [22] must be taken into account for a proper interpretation. However, a fluorescent dye, which fulfills all requirements, has not yet become available. Depending on the microscopic and experimental setup other DNA/chromatin stainings such as SYTOX Green, Vybrant or fluorescent protein-tagged histones may be chosen as well $[16,23]$.

\section{Masking of nuclei}

The space of nuclei with a fairly even shape and $z$ diameters $\leq 8 \mu \mathrm{m}$ can appropriately be assessed by automatically defining a mask as described above. We found that nucleoli contributed with $<5 \%$ to the total nuclear volume in human fibroblasts, mouse myoblast cell line $\mathrm{C} 2 \mathrm{C} 12$ and human retina epithelial-derived RPE-1 cells (data not shown) so that they can be included as part of the nucleus without special consideration. In case of huge nucleoli they should be excluded by manual cutout. A laborious manual setting of nuclear masks may also be necessary for nuclei with marked invaginations and infoldings of the nuclear lamina which can get lost by automatic mask generation. In order to achieve an optimal resolution with 3D SIM, nuclei with a flat shape and a position as close to the slide as possible are most suitable [24]. In case of very thick nuclei ( $>8 \mu \mathrm{m}$ in z-direction) [15] or nuclei with highly condensed, global chromatin compaction [16] a "glow" or "shadow" especially in the last quarter

\begin{tabular}{|l|l|l|l|l|}
\hline & $\mathbf{N}=5$ & $\mathbf{N}=7$ & $\mathbf{N = 1 1}$ & $\mathbf{N}=32$ \\
\hline marker 1 (green) & 0.01462 & 0.01005 & 0.01697 & 0.01412 \\
\hline marker 2 (red) & $2.25 e-06$ & $6.244 e-05$ & $2.41 e-06$ & $2.99 e-06$ \\
\hline marker 1 vs 2 & 0.44482 & 0.90300 & 0.31653 & 0.22526 \\
\hline
\end{tabular}

Table 1: In case of a random assignment of voxels for a functionally relevant marker (null hypothesis), the percentages of marker voxels assigned to each chromatin compaction class correspond to the percentage of DAPI intensity voxels attributed to the respective class. Table 1 shows, for example, how this null hypothesis was tested for markers 1 (top row) and 2 (middle row) attributed to 5, 7, 11 and 32 chromatin compaction classes, respectively (see Fig. 10) using the Wilcoxon test for equal distributions of voxels. The null hypothesis must be rejected in all cases on a five percent significance level. P-values for marker 1 are slightly larger because the number of signals from marker 1 is lower compared to marker 2. Bottom row: p-values for the comparison of the distribution of red and green signals indicate the same distribution of marker 1 and marker 2 on chromatin compaction classes. 
of the DAPI image stack may appear. In order to generate reasonable chromatin masks also in these sections, chromatin should be marked by hand.

\section{Number of classes for chromatin compaction levels}

Although a classification of chromatin compaction levels based on 7 DAPI intensity classes is a simplification of the actual variability of chromatin compaction levels, we chose this classification for pragmatic reasons. It yielded robust data for the assessment of compaction differences in the chromatin landscape within individual nuclei and for statistical comparisons between different nuclei and markers distributed along this landscape. Furthermore, relevant details of nuclear landscapes

A
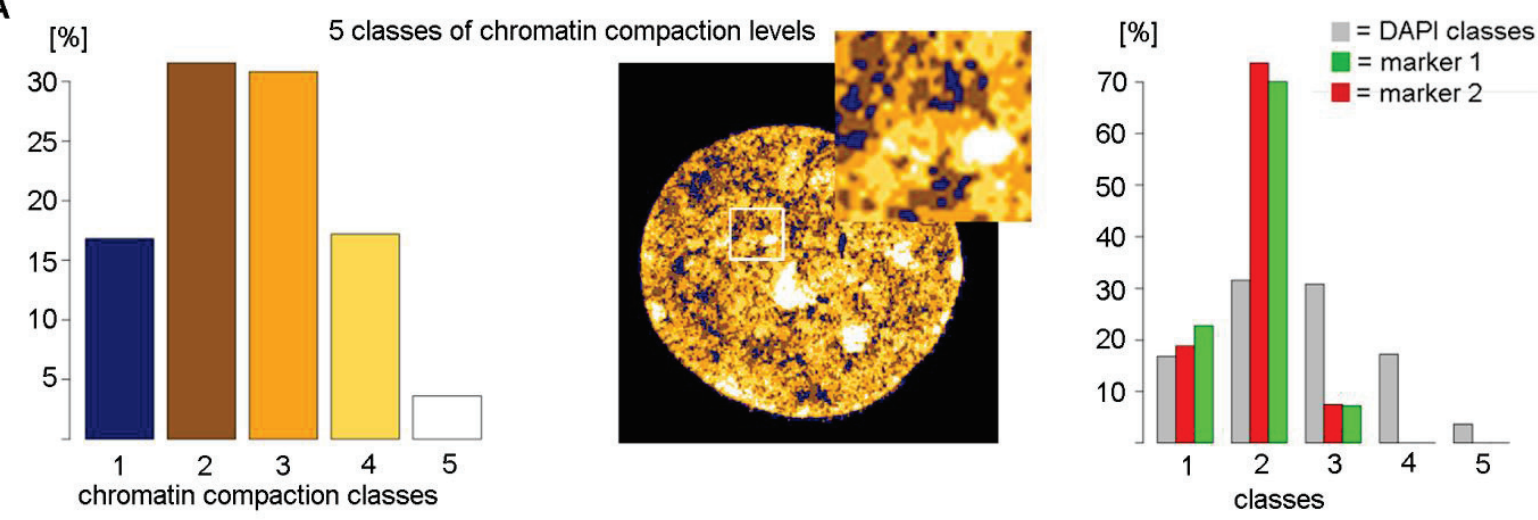

B chromatin compaction classes
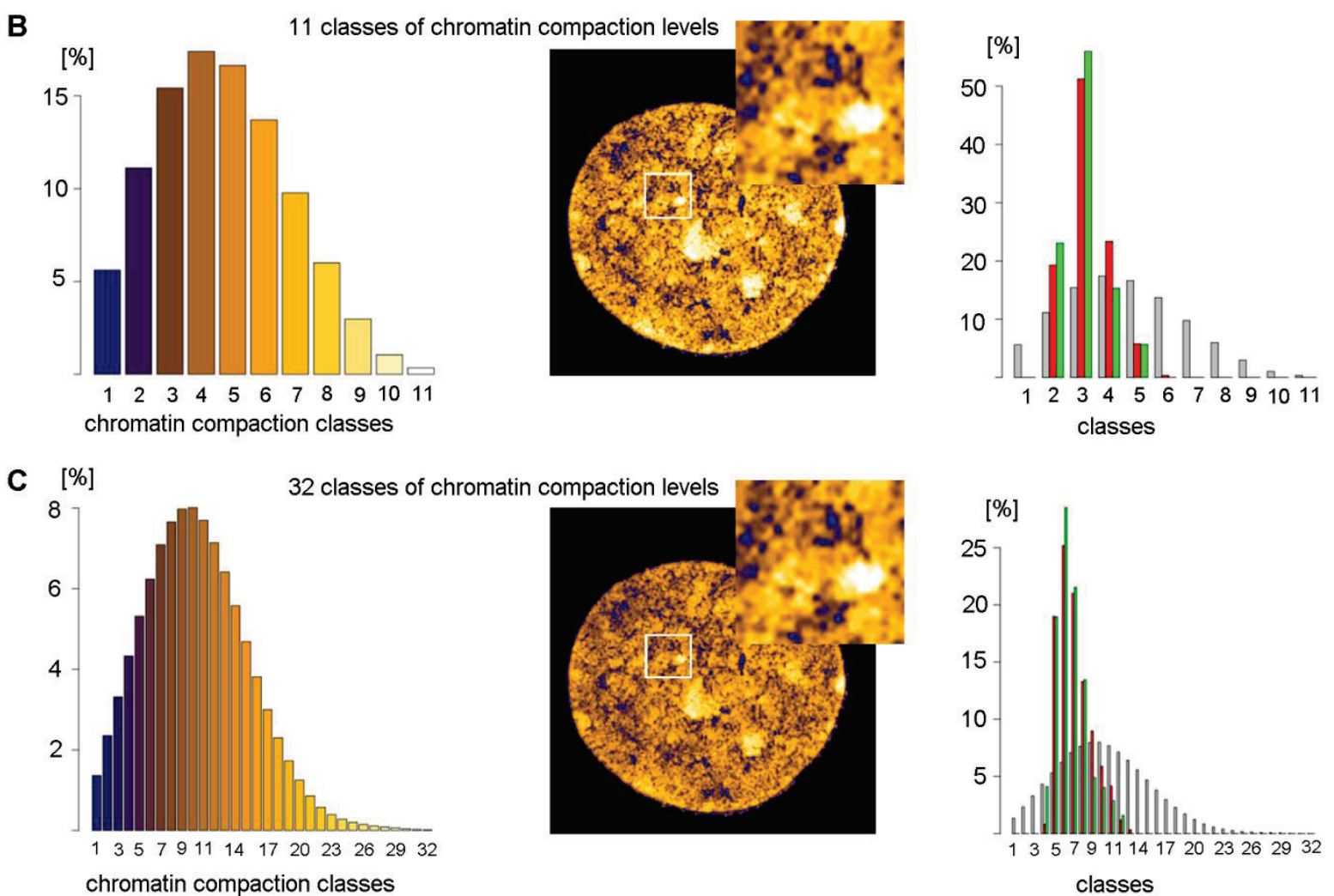

Fig. 10. Results for different numbers of chromatin compaction classes

(A) 5 classes, (B) 11 classes, (C) 32 classes. From left to right: Barplot for distribution of chromatin compaction classes, chromatin compaction map of central optical slice, distribution of red and green signal in chromatin compaction classes (using differently labeled contiguous signals as described in Fig. 7A). 
were easily noted by eye in color heat maps with a limited number of DAPI intensity classes (Figures 1C, 1E and 4). Since an increased number of classes did not affect our conclusions (see Fig. 10, Table 1), use of the theoretical limit of 65,536 grey levels in 16-bit images would increase the computational load enormously without further benefit. It should be emphasized, however, that our classification scheme tends to show borders between adjacent chromatin compaction classes, which in reality may be much fuzzier. The preferred number of classes used for statistical comparisons can of course easily be increased when such a necessity arrives, for example in case of a further gain in $3 \mathrm{D}$ resolution.

\section{Biological interpretation of DAPI intensity classes as a proxy for chromatin compaction}

Fig. 11 summarizes our current biological interpretation of the seven chromatin compaction classes in the light of the ANC-INC model of the functional nuclear organization [4].

We refer to compaction class 1 as the interchromatin compartment (IC). This concept was first introduced in electron microscopic studies of the nuclear architecture, but does not mean that DNA or chromatin loops are strictly absent within IC channels [25]. Such loops may well intrude from the lining of chromatin into the IC and the amount of such a penetration may depend on functional states and/or the cell type; compare e.g., nuclear landscapes of cultured somatic cell types [16] with blastomeres in preimplantation embryos [15] and hematopoietic cells [14]. Some important limitations of our current analytical tool should be emphasized here: 1 . While classes 1 to 7 clearly indicate increasing chromatin compaction levels, structured illumination microscopy of DAPI stained nuclei is not well suited to measure absolute differences of chromatin compaction between different classes. 2. Our approach seems suitable for comparisons of nuclear landscapes between a relatively homogeneous set of nuclei studied in the same experiment. Quantitative comparisons are much more problematic between nuclei with drastic differences in size, shape, invaginations of the nuclear envelope, higher order chromatin arrangements and absolute chromatin densities. The range of absolute compaction differences between classes 1-7 can be wider or narrower depending on seemingly subtle differences of DNA staining protocols and on thresholding of recorded image stacks. As a consequence,

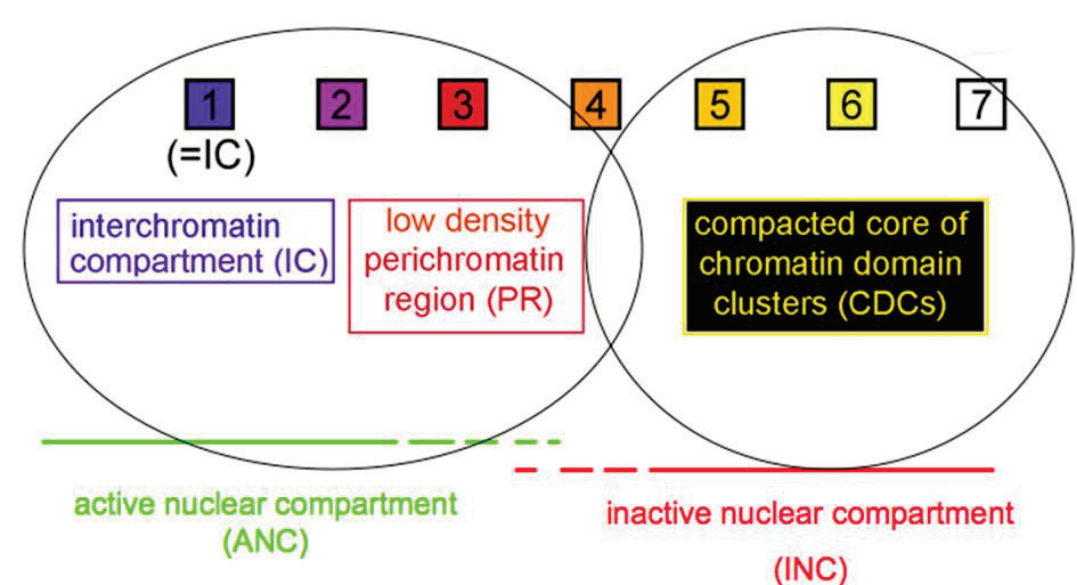

Fig. 11. Biological relevance of different chromatin compaction classes

For details see text and reference [4]. 
comparisons between, e.g., class 3 identified in one cell type with the same class identified in another cell type should be made with caution.

\section{Assessment of minimal movements of nuclear targets between regions with different chromatin compaction levels.}

A given nuclear target DNA may change its position and switch between compartments defined by different chromatin compaction levels. The relocation of a transcription regulatory element, which is present in more compact chromatin when inactive, but moves into more decondensed chromatin in response to transcriptional stimulation of target genes, may serve as an example. 3D imaging snapshots from fixed nuclei recorded at different time points after a proper stimulus applied to an entire cell population can be analyzed for a rough estimate of such positional changes, e.g. from the most compact (interior) to the most decondensed (peripheral) part of a chromatin domain cluster by measuring minimal average distances between defined chromatin compaction classes. It should, however, be emphasized that our approach can assess only minimal distances between classes, so actually travelled distances may be considerably larger.

\section{CONCLUSIONS}

The Common Fund's 4D Nucleome program of the National Institutes of Health (NIH) established in 2015 "aims to understand the principles underlying nuclear organization in space and time, the role nuclear organization plays in gene expression and cellular function, and how changes in nuclear organization affect normal development as well as various diseases." Here, we present a tool package, which may become a helpful supplement for 4D nucleome research [2]. These tools allow for a quantitative analysis of 3D image stacks from sets of fixed cell nuclei recorded with super-resolved fluorescence microscopy and are implemented as free packages in the statistical software R. They allow the assessment of chromatin compaction levels in individual cell nuclei, the measurement of distances between the chromatin compaction classes and the mapping of specific DNA / RNA sequences and nuclear proteins with respect to the 3D chromatin landscape. Statistical methods are implemented to handle noise and random artifacts in microscopic images. The mapping of specific DNA sequences and nuclear proteins on the different chromatin compaction classes allows new insights into the relationships between structural and functional processes of the 4D nucleome. Whereas the current tool package has been devised for the image analysis of the 3D nuclear landscape in fixed cells, the enhancement of these tools for the quantitative 4D image analysis of the nuclear landscape remains as a future challenge for studies of dynamic changes in in individual living cells.

\section{ACKNOWLEDGMENTS / FUNDING}

We are indebted to Lothar Schermelleh (University of Oxford, UK), Yolanda Markaki (UCLA, USA), Barbara Hübner (NTU Singapore), Daniel Smeets and Jens Popken for their support and valuable input during the development of the tool package. We gratefully acknowledge the support of the Center of Advanced Light Microcopy (CALM) of the LMU Biocenter (headed by Heinrich Leonhardt and Hartmann Harz), where all SIM images were recorded. DNA probes for the detection of sites with active and inactive regulatory sequences in A549 cell nuclei were kindly provided by John 
Stamatoyannopoulos (University of Washington, Seattle, USA). We also thank Priyanka Kukreja, Ramin Norousi and Marius Wagner for alpha testing of the tool packages.

\section{REFERENCES}

1. Sexton T, Cavalli G (2015) The Role of Chromosome Domains in Shaping the Functional Genome. Cell 160: 1049-1059.

2. Tashiro S, Lanctot C (2015) The International Nucleome Consortium. Nucleus 6: 89-92.

3. Cremer T, Cremer M (2010) Chromosome territories. Cold Spring Harb Perspect Biol 2: a003889.

4. Cremer T, Cremer M, Hubner B, Strickfaden H, Smeets D, et al. (2015) The 4D nucleome: Evidence for a dynamic nuclear landscape based on co-aligned active and inactive nuclear compartments. FEBS Lett 589: 2931-2943.

5. Chagin VO, Casas-Delucchi CS, Reinhart M, Schermelleh L, Markaki Y, et al. (2016) 4D Visualization of replication foci in mammalian cells corresponding to individual replicons. Nature Communications 7.

6. Dixon JR, Selvaraj S, Yue F, Kim A, Li Y, et al. (2012) Topological domains in mammalian genomes identified by analysis of chromatin interactions. Nature 485: 376-380.

7. Fraser J, Ferrai C, Chiariello AM, Schueler M, Rito T, et al. (2015) Hierarchical folding and reorganization of chromosomes are linked to transcriptional changes in cellular differentiation. Mol Syst Biol 11: 852.

8. Godin AG, Lounis B, Cognet L (2014) Super-resolution microscopy approaches for live cell imaging. Biophysical Journal 107: 1777-1784.

9. Long F, Zhou J, Peng H (2012) Visualization and Analysis of 3D Microscopic Images. PLoS Comput Biol 8(6): e1002519. doi:1002510.1001371/journal.pcbi.1002519.

10. Zhang Y, Brady M, Smith S (2001) Segmentation of brain MR images through a hidden Markov random field model and the expectation-maximization algorithm. IEEE Trans Med Imaging 20: 45-57.

11. Lei T (2012) Statistics of Medical Imaging. Pittsburgh:CRC press.

12. R Core Team (2016) R: A language and environment for statistical computing. R Foundation for Statistical Computing, Vienna, Austria.

13. R Studio Team (2016) RStudio: Integrated Development for R. RStudio, Inc., Boston, MA

14. Hubner B, Lomiento M, Mammoli F, Illner D, Markaki Y, et al. (2015) Remodeling of nuclear landscapes during human myelopoietic cell differentiation maintains co-aligned active and inactive nuclear compartments. Epigenetics Chromatin 8: 47.

15. Popken J, Brero A, Koehler D, Schmid VJ, Strauss A, et al. (2014) Reprogramming of fibroblast nuclei in cloned bovine embryos involves major structural remodeling with both striking similarities and differences to nuclear phenotypes of in vitro fertilized embryos. Nucleus 5: 555-589.

16. Smeets D, Markaki Y, Schmid VJ, Kraus F, Tattermusch A, et al. (2014) Three-dimensional superresolution microscopy of the inactive $X$ chromosome territory reveals a collapse of its active nuclear compartment harboring distinct Xist RNA foci. Epigenetics Chromatin 7: 8.

17. Schermelleh L, Heintzmann R, Leonhardt H (2010) A guide to super-resolution fluorescence microscopy. J Cell Biol 190: 165-175.

18. Ball G, Demmerle J, Kaufmann R, Davis I, Dobbie IM, et al. (2015) SIMcheck: a Toolbox for Successful Super-resolution Structured Illumination Microscopy. Sci Rep 5: 15915.

19. Pau G, Fuchs F, Sklyar O, Boutros M, Huber W (2010) EBlmage-an R package for image processing with applications to cellular phenotypes. Bioinformatics 26: 979-981.

20. Carpenter AE, Jones TR, Lamprecht MR, Clarke C, Kang IH, et al. (2006) CellProfiler: image analysis software for identifying and quantifying cell phenotypes. Genome Biol 7: R100.

21. Bolte S, Cordelieres E (2006) A guided tour into subcellular colocalization analysis in light microscopy. Journal of Microscopy: 224.

22. Chazotte B (2011) Labeling Nuclear DNA Using DAPI. Cold Spring Harb protoc doi:10.1101/pdb.prot5556.

23. Szczurek AT, Klewes, L, Xing, J, Gourram, A, Birk, U, Knecht, H, Dobrucki, JW, Mai, S, Cremer, C (2017) Imaging chromatin nanostructures with binding-activated localization micropscopy based on DNA structure fluctuations. Nucleic Acid Res gkw1301.

24. Popken J, Dahlhoff M, Guengoer T, Wolf E, Zakhartchenko V (2015) 3D structured illumination microscopy of mammalian embryos and spermatozoa. BMC Dev Biol 15: 46.

25. Rouquette J, Cremer C, Cremer T, Fakan S (2010) Functional nuclear architecture studied by microscopy: present and future. Int Rev Cell Mol Biol 282: 1-90. 